\title{
Characterization of Motor Neuron Prostaglandin E2 EP3 Receptor Isoform in a Mouse Model of Amyotrophic Lateral Sclerosis
}

\author{
Yasuhiro Kosuge, ${ }^{*, a, b, \#}$ Hiroko Miyagishi, ${ }^{a, c, \#}$ Takashi Shinomiya, ${ }^{b}$ Kenta Nishiyama, ${ }^{a}$ \\ Satomi Suzuki, ${ }^{a}$ Nobuhiro Osada,${ }^{a}$ Kumiko Ishige, ${ }^{a}$ Migiwa Okubo, ${ }^{b}$ Mitsuru Kawaguchi, ${ }^{b}$ and \\ Yoshihisa Ito ${ }^{a}$ \\ ${ }^{a}$ Laboratory of Pharmacology, School of Pharmacy, Nihon University; 7-7-1 Narashinodai, Funabashi, Chiba \\ 274-8555, Japan: ${ }^{b}$ Department of Pharmacology, Tokyo Dental College; 2-9-18 Misaki-cho, Chiyoda-ku, Tokyo \\ 101-0061, Japan: and ${ }^{c}$ Department of Pharmacology, School of Pharmacy, International University of Health and \\ Welfare; 2600-1 Kitakanemaru, Ohtawara, Tochigi 324-8501, Japan.
}

Received May 15, 2015; accepted September 5, 2015

Amyotrophic lateral sclerosis (ALS) is a motor neuron disease with adult onset, characterized by progressive loss of motor neurons. Prostaglandin E2 (PGE2), a lipid mediator, exerts its biological functions by binding to four subtypes of E-prostanoid (EP1-4). Among them, EP3 has been shown to have multiple isoforms, EP3 $\alpha, \mathrm{EP} \beta \beta$, and EP3 $\gamma$, produced by alternative splicing. Since PGE2 has been shown to have important pathophysiological roles in ALS, experiments were performed to identify EP3 receptor isoform(s) in spinal motor neurons of wild-type (WT) and ALS model (G93A) mice. Reverse transcription-polymerase chain reaction (RT-PCR) analysis of adult mice demonstrated expression of EP3 $\alpha$ and EP3 $\gamma$ mNAs in the lumbar spinal cord, whereas EP3 $\beta$ mRNA was barely detectable. Laser capture microdissection was used to dissect out motor neurons from frozen samples of lumbar spinal cord in these mice for analysis by real-time PCR. We found that expression of EP3 $\gamma$ mRNA was predominant in these neurons, whereas EP3 $\alpha$ and EP3 $\beta$ mRNAs were undetectable. At the early symptomatic stage, the mRNA expression profiles of these splice isoforms in G93A motor neurons were comparable to those in neurons from WT mice. These results suggest that the PGE2-to-EP3 signaling pathway is mediated mainly by the EP3 $\gamma$ isoform in the motor neurons of mice, and that modulation of the EP3 $\gamma$ isoform in motor neurons may be a promising new therapeutic approach for ALS.

Key words motor neuron; prostaglandin E2 (PGE2); E-prostanoid 3 (EP3) receptor isoform; amyotrophic lateral sclerosis; laser microdissection

Amyotrophic lateral sclerosis (ALS) is a devastating progressive neurodegenerative disease characterized by selective loss of motor neurons in the cortex and spinal cord. ALS causes progressive muscular paralysis reflecting the degeneration of motor neurons, a poor quality of life, and eventual death within 3-5 years of diagnosis. ${ }^{1)}$ Although multiple processes are thought to be implicated in the pathogenesis of ALS, including oxidative stress, mitochondrial dysfunction, excitotoxicity, RNA-processing errors and abnormal protein aggregation, ${ }^{2)}$ the mechanism underlying the selective death of motor neurons has not yet been clarified. Recently, inflammation in the spinal cord has been highlighted as an important pathogenic mechanism in ALS, and levels of prostaglandin E2 (PGE2), a key proinflammatory mediator, are increased in postmortem brain tissue, cerebrospinal fluid and serum from patients with sporadic ALS. ${ }^{3,4)}$ It has also been shown that ALS model mice have markedly increased levels of PGE2 in both the cerebral cortex and spinal cord. ${ }^{5)}$ Moreover, the expression of cyclooxygenase (COX)-2, which plays a key role in the synthesis of prostaglandins from arachidonic acid, is up-regulated in the spinal cord of ALS patients and model mice. ${ }^{6,7)}$ Treatment with celecoxib, a selective COX-2 inhibitor, delays the onset of the disease and prolongs survival. ${ }^{8} \mathrm{Re}$ cently, we have also shown that the level of microsomal PGE synthase-1 (mPGES-1), catalyzing the terminal step of PGE2

\footnotetext{
\# These authors contributed equally to this work.

biosynthesis, was significantly increased in the motor neurons of ALS model mice. ${ }^{9}$ Furthermore, inhibition of mPGES-1 by AAD-2004, a dual-function drug derived from aspirin and sulfasalazine, prevented the elevation of PGE2 content in the spinal cord of ALS model mice, and improved both motor function and survival. ${ }^{10)}$ These results suggest that PGE2 plays a pivotal role in the pathogenesis of ALS.

PGE2 activates four specific $G$ protein-coupled E prostanoid (EP) receptors $(E P 1-4) .{ }^{11)}$ It has been reported that EP1 is involved in the regulation of intracellular $\mathrm{Ca}^{2+}$ levels, that EP2 and EP4 increase the intracellular levels of cAMP through activation of adenyl cyclase via stimulatory G (Gs) protein, and that EP3 decreases or increases intracellular cAMP levels by activating inhibitory $\mathrm{G}$ (Gi) protein or Gs protein, depending on the specialized splice variants. ${ }^{11)}$ The murine EP3 receptor consists of three distinct isoforms produced by alternative splicing of the cytoplasmic carboxy-terminal tail. ${ }^{11)}$ The EP $3 \alpha$ and EP3 $\beta$ isoforms are exclusively coupled to Giprotein, whereas the EP $3 \gamma$ isoform is coupled to both Gs- and Gi-protein. ${ }^{11)}$ More recently, we revealed that EP2 and EP3 were expressed predominantly at the protein level in mouse spinal motor neurons and NSC-34 cells, a motor neuron-like cell line, and that EP2 plays a crucial role in PGE2-induced cytotoxicity in NSC-34 cells. ${ }^{12)}$ However, the specific role of the EP3 isoform in motor neurons is poorly understood. In the present study we aimed to characterize the EP3 receptor isoforms in motor neurons of the mouse spinal cord, and to 
clarify the influence of isoform distribution on the onset and progression of ALS.

\section{MATERIALS AND METHODS}

Animal Transgenic male mice overexpressing mutated (glycine to alanine in position 93) human SOD1 ${ }^{\mathrm{G} 93 \mathrm{~A}}$ (G93A mice) were used as a mouse model of ALS (Jackson Laboratory, Bar Harbor, ME, U.S.A.). The hemizygous G93A mice were maintained by mating transgenic males with wild-type females. G93A and wild-type (WT) mice were housed under standard conditions (temperature $22^{\circ} \mathrm{C}$, relative humidity $60 \%$, $12 \mathrm{~h}$ light/dark cycle, and free access to food and water) in the animal facility at the School of Pharmacy, Nihon University. Genotyping was performed using genomic DNA extracted from tails by polymerase chain reaction (PCR) as reported previously. ${ }^{9}$ All efforts were made to minimize the number of animals used and their distress. All experiments with animals complied with the Guidelines for Animal Experiments at Nihon University.

Histological Analysis Anesthetized animals were perfused with $4 \%$ paraformaldehyde in $0.1 \mathrm{~m}$ phosphate buffer ( $\mathrm{pH}$ 7.4). Lumbar spinal cord were removed and post fixed for $24 \mathrm{~h}$ in the same fixative. Post-fixed spinal cord were embedded in Optimal Tissue Compound and cut with a cryostat into $20-\mu \mathrm{m}$ sections. The spinal cord sections were stained with Cresyl Violet. Motor neurons were defined according to the following three criteria: (i) Nissl-stained cell; (ii) localization in ventral horns; (iii) diameter $>25 \mu \mathrm{m}$.

Laser Microdissection (LMD) The cryo-sections (20$\mu \mathrm{m})$ were fixed with $100 \%$ ethanol for $60 \mathrm{~s}$ and air-dried. Following toluidine blue staining, motor neurons in lumbar spinal cord were dissected out from the slices and collected using a LMD (P.A.L.M. Microlaser Technologies, Bernried, Germany). ${ }^{13,14)}$ The motor neurons collected by LMD (approximately 200 cells) were placed on PALM Adhesive Caps (P.A.L.M. Microlaser Technologies), immediately lysed in Lysis/Binging buffer (Roche Diagnostics, GmgH, Penzberg, Germany), and stored at $-80^{\circ} \mathrm{C}$ until total RNA extraction.

Extraction of Total RNA and Real-Time Semi-quantitative PCR Analysis Gene expression of the EP3 isoforms was determined by semi-quantitative real-time reverse transcription (RT)-PCR as previously described with modifica- tions. ${ }^{15)}$ Briefly, total RNA was extracted from frozen spinal cord tissues and microdissected motor neurons with the High Pure RNA Isolation kit (Roche Diagnostics) according to the manufacturer's instructions. After DNase treatment with DNase I, first-strand cDNA was synthesized by using Oligo $(\mathrm{dT})_{18}$ primers and Transcriptor First Strand cDNA Synthesis Kit (Roche Diagnostics). Aliquots of cDNA were amplified on a MX3000P real-time PCR system (Stratagene, La Jolla, CA, U.S.A.) using SYBR ${ }^{\circledR}$ Premix Ex Taq ${ }^{\mathrm{TM}}$ II (TaKaRa Biotechnology, Shiga, Japan). The forward and reverse primer sets used are listed in Table 1. ${ }^{16)}$ All samples were assayed in triplicate. All data were normalized to an internal standard ( $\beta$-actin; $\triangle \Delta$ CT method).

Statistical Analysis All the data are expressed as the mean \pm standard error of the mean (S.E.M.). Statistical significance was assessed by a paired Student's $t$-test. $p$-Values of $<0.05$ were considered statistically significant.

\section{RESULTS AND DISCUSSION}

The motor neuron degeneration and symptoms of ALS are progressive and age-dependent in both patients and rodent models. Recently, we have reported that in G93A mice agedependent decreases in motor performance begin at the early symptomatic stage (14 and 15 weeks of age), followed by a decrease in immunoreactivity for SMI32, a neuronal marker protein, at 17 and 19 weeks. ${ }^{9)}$ As in the case of SMI32, the number of large motor neurons in G93A mice was not significantly different from that in WT mice at 15 weeks of age (Fig. 1). These results are consistent with previous results shown that the number of motor neurons in G93A tended to decrease

Table 1. Primer Sequences for Real-Time RT-PCR

\begin{tabular}{lc}
\hline \multicolumn{1}{c}{ Gene } & Primer sequences $\left(5^{\prime} \rightarrow 3^{\prime}\right)$ \\
\hline EP3 $\alpha$ forward & CGG AAG TTC TGC CAG ATC AGA \\
EP3 $\alpha$ reverse & TCC AGC TGG TCA CTC CAC ATC \\
EP3 $\beta$ forward & CGG AAG TTC TGC CAG ATG ATG \\
EP3 $\beta$ reverse & CAG GGA AAC AGG TAC TGC AAT G \\
EP3 $\gamma$ forward & AGT TCT GCC AGG TAG CAA ACG \\
EP3 $\gamma$ reverse & GCC TGC CCT TTC TGT CCA T \\
$\beta$-Actin forward & CAT CCG TAA AGA CCT CTA TGC CAA C \\
$\beta$-Actin reverse & ATG GAG CCA CCG ATC CAC A \\
\hline
\end{tabular}

(A) Nissle stain

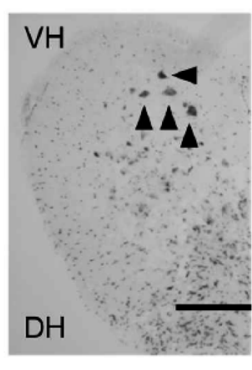

WT

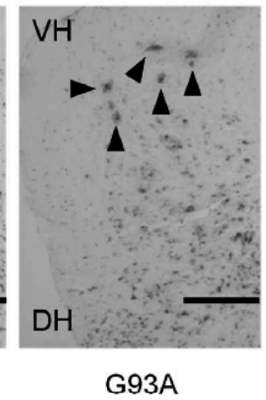

(B) Motor neuron

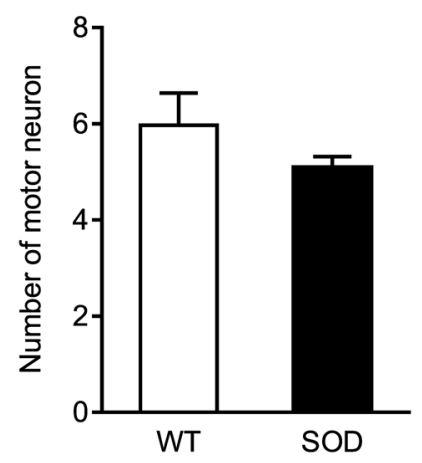

Fig. 1. Histological Analysis of Lumbar Spinal Cord Tissue in WT and G93A Mice

(A) Representative cresyl violet-stained sections of the lumbar spinal cord in WT (left) and G93A mice (right) at 15 weeks of age. Arrowheads indicate motor neurons. Scale bar indicates $100 \mu \mathrm{m}$. DH; dorsal horn. VH; ventral horn. (B) Graphs show numbers of surviving motor neurons in lumbar spinal cord sections from WT and G93A mice. Values represent the mean \pm S.E.M., $n=5$. 
at the early symptomatic stage, but there was no significant difference in the number of the cells between G93A and WT mice. ${ }^{17)}$ On the other hand, some groups reported that the number of motor neuron decreased significantly in G93A mice at 15 weeks of age. ${ }^{18,19)}$ Taken together, these data suggested that the characteristics of the motor neurons depend on each G93A mice even carry the same SOD1 mutations. One reason for this variation might be attributable to a major quantitative trait locus (QTL) on mouse chromosome 17 that affect the lifespan in G93A mice. ${ }^{20)}$ We also demonstrated a significant increase of mPGES-1, catalyzing the terminal step of PGE2 biosynthesis, in motor neurons of the ventral horn at 15 weeks of age in G93A mice. ${ }^{9)}$ Since we wished to focus on neuronal changes associated with the EP-mediated signaling pathway at the early symptomatic stage, we investigated the changes in the levels of EP3 isoforms in the anterior horn of the spinal cord, and in motor neurons, at 15 weeks of age.

First, we quantified the levels of mRNA expression for all of the EP3 isoforms in G93A and WT mice in the lumbar spinal cord at 15 weeks. Real-time PCR demonstrated expression of both EP $3 \alpha$ and EP $3 \gamma$ mRNAs in both groups, but there were no differences in the levels of EP3 $\alpha$ and EP3 $\gamma$ mRNAs between G93A and WT mice (Fig. 2). In contrast, EP3 $\beta$ mRNA was barely detectable in both groups. These results were consistent with a previous study revealing that the EP3 $\alpha$ and EP $3 \gamma$ isoforms are dominantly expressed in mouse spinal cord tissue. ${ }^{21)}$ Earlier studies from other laboratories had shown that all of the EP3 receptor splice variants are ubiquitously expressed in eight major regions of the adult mouse brain: the cortex, frontal cortex, thalamus, hypothalamus, hippocampus, brainstem, medulla and cerebellum. ${ }^{16)}$ Unlike these brain regions, not all of the EP3 receptor splice variants were expressed in the mouse spinal cord, the EP3 $\alpha$ and EP3 $\gamma$ isoforms playing roles in PGE2-mediated functions in this location.

Next, we sought to identify EP3 receptor isoforms in motor neurons of the spinal cord in both G93A and WT mice using laser capture microdissection. RNA extracted from isolated motor neurons from frozen sections of the lumbar spinal cord (Fig. 3A) was examined to quantify the expressed EP3 splice isoforms (Fig. 3B). Semi-quantitative PCR revealed predominant expression of EP3 $\gamma$ in motor neurons, whereas EP3 $\alpha$ was not detected in either G93A or WT mice. Moreover, at the early symptomatic stage, the level of EP3 $\gamma$ expression in motor neurons of G93A mice was comparable to that in WT mice (Figs. 2, 3). These results suggest that EP $3 \gamma$, but not EP $3 \alpha$ or $\mathrm{EP} 3 \beta$, is predominantly expressed and functions in PGE2-EP3 signaling in mouse motor neurons, implying that alternative splicing of the EP3 receptor does not change during the onset and/or progression of ALS. In the mouse preoptic region of the anterior hypothalamus, the expression profile of EP3 variants was reportedly not changed by immune challenge induced by systemic injection of lipopolysaccharide. ${ }^{22)}$ Taken together, these findings suggest that EP3 receptor splicing is not activity-dependent in the spinal cord or the hypothalamus. Although the alternatively spliced EP3 receptor isoforms

\section{EP3 isoform (15 week)}

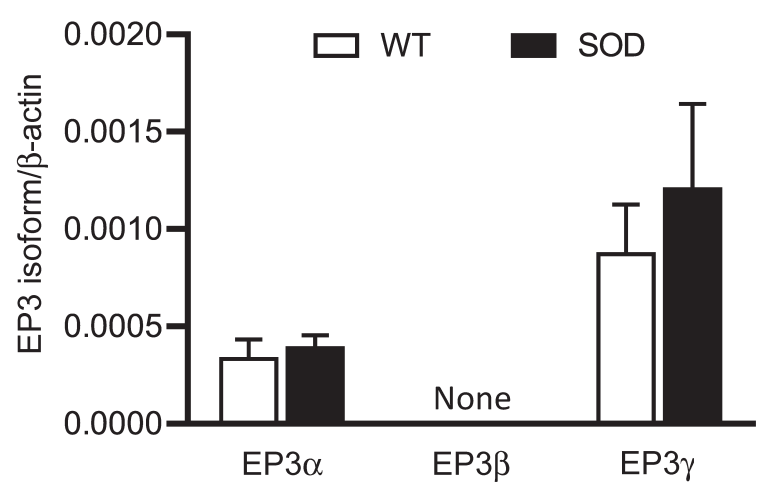

Fig. 2. Characterization of EP3 Isoform mRNA Expression in the Lumbar Spinal Cord of WT and G93A Mice

Graphs show the expression profiles of mRNAs for EP3 splice isoforms in the lumbar spinal cord of WT and G93A mice at 15 weeks of age. Expression of mRNA for each EP3 isoform was normalized to the level of $\beta$-actin mRNA. Values represent the mean \pm S.E.M., $n=5$.
(A) LMD
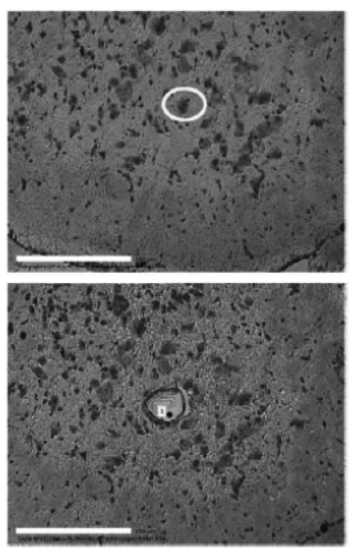

(B) EP3 isoform in motor neuron

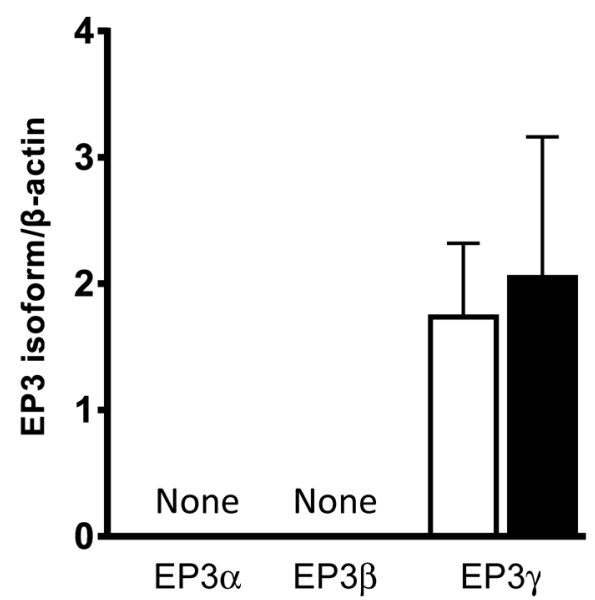

Fig. 3. Distribution Pattern of EP3 Isoforms in Motor Neurons of the Lumbar Spinal Cord of WT and G93A Mice

(A) Dissection of motor neurons from fresh frozen mouse spinal cord sections using a LMD system. Photograph of representative toluidine blue-stained sections before dissection (upper panel) and after (lower panel) microdissection. A circle indicates the collected motor neurons in the anterior horns of the spinal cord. Scale bar indicates $150 \mu \mathrm{m}$. (B) Graphs show the expression profiles of mRNAs for EP3 splice isoforms in the motor neurons of WT and G93A mice at 15 weeks of age. Expression of mRNA for each EP3 isoform was normalized to the level of $\beta$-actin mRNA. Values represent the mean \pm S.E.M., $n=5$. 
have similar PGE2 binding properties, they differ in their specificity of coupling to G-proteins. ${ }^{11)}$ The EP3 $\alpha$ and EP3 $\beta$ isoforms are couple exclusively to Gi-protein, ${ }^{23,24)}$ whereas the EP $3 \gamma$ isoform shows mostly constitutive Gi activity and agonist-dependent Gs activity. ${ }^{25)}$ Because no selective Gsactivating ligand of the EP3 $\gamma$ isoform is commercially available, little information is available about the effect of activation of the EP3 $\gamma$ isoform on neurodegeneration and neuronal damage in ALS model mice.

A previous study conducted in our laboratory showed that EP2, a Gs-coupled receptor, plays a key role in PGE2-induced death of motor-neuron-like NSC-34 cells. ${ }^{12)}$ This suggests that activation of Gs-protein via the EP3 $\gamma$ receptor may also be involved in PGE2-induced neurotoxicity in spinal cord motor neurons in ALS model mice. These findings, together with previous results, suggest that, in ALS model mice, elevation of the PGE2 level by up-regulation of mPGES-19) induces motor neuronal death through activation of Gs-protein-coupled EP receptors such as EP2 and EP3 $\gamma$.

On the other hand, a previous study using rat organotypic spinal cord slice cultures as an in vitro model of ALS showed that application of an EP3 agonist, sulprostone, paradoxically protected motor neurons from chronic glutamate-induced neuronal degeneration via Gi-protein-coupled signaling. ${ }^{26)}$ As shown in the present and previous studies, ${ }^{21)}$ the EP $3 \alpha$ and $\mathrm{EP} 3 \gamma$ isoforms are expressed predominantly in mouse spinal cord tissue, and the EP3 $\alpha$ isoform, a Gi-protein-coupled receptor, was not detected in motor neurons. Therefore, the neuroprotective effect against chronic glutamate-induced neuronal degeneration via activation of the EP3 receptor might be attributable to activation of the EP $3 \alpha$ isoform in non-neuronal cells such as astrocytes and microglia. As previously observed, cannabinoid receptor type1 (CB1), a Gi-protein-coupled receptor, is present and expressed in mouse spinal cord neurons and glial cells. ${ }^{27)}$ Furthermore, activation of the CB1 protects cultured mouse spinal neurons against kainate-induced excitotoxicity. ${ }^{27)}$ Thus, there is a possibility that activation of Gi-protein via the EP3 $\gamma$ receptor in motor neuron will show the neuroprotective effect. Further studies will be necessary to clarify the dual effects elicited by the EP3-mediated pathway on motor neuron survival in ALS models.

In conclusion, we have demonstrated for the first time that the EP $3 \gamma$ isoform is expressed predominantly in mouse spinal motor neurons showing elevation of PGE2 biosynthesis at the early symptomatic stage. Although the expression profile of EP3 isoforms in motor neurons is not altered by the onset of ALS, modulation of the EP $3 \gamma$ isoform by specific drugs could be a novel therapeutic strategy for targeting the $\mathrm{PGE}_{2}$ receptor pathways in models of ALS.

Acknowledgments We thank K. Tadokoro (T.D.C.) for technical assistance. This work was supported by a Grant from the Takeda Science Foundation (Y.K.), a Grant for novel research project in the School of Pharmacy Nihon University (Y.K.), and a Grant-in-Aid for Challenging Exploratory Research (15K14966 to Y.I.) from Japan Society for the Promotion of Science (JSPS).

Conflict of Interest The authors declare no conflict of interest.

\section{REFERENCES}

1) Wijesekera LC, Leigh PN. Amyotrophic lateral sclerosis. Orphanet J. Rare Dis., 4, 3 (2009).

2) Ferraiuolo L, Kirby J, Grierson AJ, Sendtner M, Shaw PJ. Molecular pathways of motor neuron injury in amyotrophic lateral sclerosis. Nat. Rev. Neurol., 7, 616-630 (2011).

3) Almer G, Teismann P, Stevic Z, Halaschek-Wiener J, Deecke L, Kostic V, Przedborski S. Increased levels of the pro-inflammatory prostaglandin PGE2 in CSF from ALS patients. Neurology, 58, 1277-1279 (2002).

4) Iłżecka J. Prostaglandin E2 is increased in amyotrophic lateral sclerosis patients. Acta Neurol. Scand., 108, 125-129 (2003).

5) Klivenyi P, Kiaei M, Gardian G, Calingasan NY, Beal MF. Additive neuroprotective effects of creatine and cyclooxygenase 2 inhibitors in a transgenic mouse model of amyotrophic lateral sclerosis. $J$. Neurochem., 88, 576-582 (2004).

6) Almer G, Guégan C, Teismann P, Naini A, Rosoklija G, Hays AP, Chen C, Przedborski S. Increased expression of the pro-inflammatory enzyme cyclooxygenase-2 in amyotrophic lateral sclerosis. Ann. Neurol., 49, 176-185 (2001).

7) Yasojima K, Tourtellotte WW, McGeer EG, McGeer PL. Marked increase in cyclooxygenase-2 in ALS spinal cord: implications for therapy. Neurology, 57, 952-956 (2001).

8) Drachman DB, Frank K, Dykes-Hoberg M, Teismann P, Almer G, Przedborski S, Rothstein JD. Cyclooxygenase 2 inhibition protects motor neurons and prolongs survival in a transgenic mouse model of ALS. Ann. Neurol., 52, 771-778 (2002).

9) Miyagishi H, Kosuge $\mathrm{Y}$, Ishige K, Ito Y. Expression of microsomal prostaglandin E synthase-1 in the spinal cord in a transgenic mouse model of amyotrophic lateral sclerosis. J. Pharmacol. Sci., 118, 225-236 (2012).

10) Shin JH, Lee YA, Lee JK, Lee YB, Cho W, Im DS, Lee JH, Yun BS, Springer JE, Gwag BJ. Concurrent blockade of free radical and microsomal prostaglandin E synthase-1-mediated PGE2 production improves safety and efficacy in a mouse model of amyotrophic lateral sclerosis. J. Neurochem., 122, 952-961 (2012).

11) Sugimoto Y, Narumiya S. Prostaglandin E receptors. J. Biol. Chem., 282, 11613-11617 (2007).

12) Miyagishi H, Kosuge $\mathrm{Y}$, Yoneoka $\mathrm{Y}$, Ozone M, Endo M, Osada N, Ishige K, Kusama-Eguchi K, Ito Y. Prostaglandin E2-induced cell death is mediated by activation of EP2 receptors in motor neuronlike NSC-34 cells. J. Pharmacol. Sci., 121, 347-350 (2013).

13) Kosuge $Y$, Imai $T$, Kawaguchi M, Kihara T, Ishige K, Ito Y. Subregion-specific vulnerability to endoplasmic reticulum stress-induced neurotoxicity in rat hippocampal neurons. Neurochem. Int., 52, 1204-1211 (2008).

14) Sobhan U, Sato M, Shinomiya T, Okubo M, Tsumura M, Muramatsu T, Kawaguchi M, Tazaki M, Shibukawa Y. Immunolocalization and distribution of functional temperature-sensitive TRP channels in salivary glands. Cell Tissue Res., 354, 507-519 (2013).

15) Oda T, Kosuge Y, Arakawa M, Ishige K, Ito Y. Distinct mechanism of cell death is responsible for tunicamycin-induced ER stress in SK-N-SH and SH-SY5Y cells. Neurosci. Res., 60, 29-39 (2008).

16) Tamiji J, Crawford DA. Prostaglandin $\mathrm{E}_{2}$ and misoprostol induce neurite retraction in Neuro-2a cells. Biochem. Biophys. Res. Commun., 398, 450-456 (2010).

17) Tönges L, Gunther R, Suhr M, Jansen J, Balck A, Saal KA, Barski E, Nientied T, Götz AA, Koch JC, Mueller BK, Weishaupt JH, Sereda MW, Hanisch UK, Bähr M, Lingor P. Rho kinase inhibition modulates microglia activation and improves survival in a model of amyotrophic lateral sclerosis. Glia, 62, 217-232 (2014).

18) Zhao W, Varghese M, Yemul S, Pan Y, Cheng A, Marano P, Hassan S, Vempati P, Chen F, Qian X, Pasinetti GM. Peroxisome proliferator activator receptor gamma coactivator-1alpha (PGC-1 $\alpha$ ) improves motor performance and survival in a mouse model of amyotrophic 
lateral sclerosis. Mol. Neurodegener., 6, 51 (2011).

19) Huang G, Lee X, Bian Y, Shao Z, Sheng G, Pepinsky RB, Mi S. Death receptor 6 (DR6) antagonist antibody is neuroprotective in the mouse SOD1 ${ }^{\mathrm{G} 93 \mathrm{~A}}$ model of amyotrophic lateral sclerosis. Cell Death Dis., 4, e841 (2013).

20) Sher RB, Heiman-Patterson TD, Blankenhorn EA, Jiang J, Alexander G, Deitch JS, Cox GA. A major QTL on mouse chromosome 17 resulting in lifespan variability in SOD1-G93A transgenic mouse models of amyotrophic lateral sclerosis. Amyotroph Lateral Scler. Frontotemporal Degener., 15, 588-600 (2014).

21) Popp L, Haussler A, Olliges A, Nusing R, Narumiya S, Geisslinger $\mathrm{G}$, Tegeder I. Comparison of nociceptive behavior in prostaglandin E, F, D, prostacyclin and thromboxane receptor knockout mice. Eur. J. Pain, 13, 691-703 (2009).

22) Vasilache AM, Andersson J, Nilsberth C. Expression of $\mathrm{PGE}_{2} \mathrm{EP}_{3}$ receptor subtypes in the mouse preoptic region. Neurosci. Lett., 423, 179-183 (2007).
23) Hasegawa $H$, Negishi M, Ichikawa A. Two isoforms of the prostaglandin E receptor EP3 subtype different in agonist-independent constitutive activity. J. Biol. Chem., 271, 1857-1860 (1996).

24) Irie A, Sugimoto $Y$, Namba T, Asano T, Ichikawa A, Negishi M. The C-terminus of the prostaglandin-E-receptor $\mathrm{EP}_{3}$ subtype is essential for activation of GTP-binding protein. Eur. J. Biochem., 224, 161-166 (1994).

25) Negishi M, Hasegawa H, Ichikawa A. Prostaglandin E receptor EP3 $\gamma$ isoform, with mostly full constitutive Gi activity and agonistdependent Gs activity. FEBS Lett., 386, 165-168 (1996).

26) Bilak M, Wu L, Wang Q, Haughey N, Conant K, St Hillaire C, Andreasson $\mathrm{K}$. $\mathrm{PGE}_{2}$ receptors rescue motor neurons in a model of amyotrophic lateral sclerosis. Ann. Neurol., 56, 240-248 (2004).

27) Abood ME, Rizvi G, Sallapudi N, McAllister SD. Activation of the $\mathrm{CB}_{1}$ cannabinoid receptor protects cultured mouse spinal neurons against excitotoxicity. Neurosci. Lett., 309, 197-201 (2001). 